\title{
Las yeserías del Patio de las Doncellas del Real Alcázar de Sevilla. Avance de resultados de la caracterización de materiales y la técnica
}

\author{
The Plasterwork at Courtyard of the Maidens in the \\ Alcazar of Seville. Initial Contributions of \\ the Characterisation of Materials and Techniques
}

\author{
Ana Isabel Calero-Castillo \\ Ana García-Bueno \\ Olimpia López-Cruz \\ Víctor Jesús Medina-Flórez \\ Universidad de Granada
}

Este artículo presenta un avance de resultados del estudio de la policromía de las yeserías del Patio de las Doncellas del Real Alcázar de Sevilla. Este espectacular patio es parte del Palacio de Pedro I (1356- 1366), considerado como una de las obras mudéjares de mayor relevancia y uno de los palacios más antiguos todavía en uso. Su condición continuada como residencia de los monarcas españoles, ha permitido su conservación, pero también ha supuesto numerosas intervenciones que se agudizan en las policromías de los revestimientos decorativos.

Tras un completo trabajo de campo se realizó un muestreo de la zona con el objetivo de conocer la naturaleza de los materiales e identificar las intervenciones realizadas. Para ello se han utilizado diversas técnicas analíticas (microscopía óptica, microscopía electrónica de barrido SEM/EDX, métodos cromatográficos y difracción de rayos X). Los resultados han per-
This article presents the initial contributions of the plasterworks polychrome research carried out at Courtyard of the Maidens in the Alcazar of Seville. This spectacular courtyard is located within the King Pedro I Palace (1356-1366), which is considered one of the most representative buildings mudejar architecture. This palace has been one of the residences of the Spanish monarchs over the time, thus it is one of the oldest palaces still in use. Consequently many interventions took place in the building, especially on the polychrome decoration.

As part of the field-work, samples were collected aiming to identify the different polychrome interventions and the materials used in each of them. Different techniques were used to analyse the samples (optical microscopy, electron microscopy SEM / EDX, chromatographic methods and X-ray diffraction). The results of the study confirmed that successive 
mitido confirmar la existencia de policromías sucesivas sobre la obra original. En los estratos más profundos, y por lo tanto probablemente originales, se han identificado restos de pigmentos naturales como rojo de plomo, cinabrio, azurita natural o malaquita, mientras que en los estratos más superficiales se identifican azul ultramar artificial o verde esmeralda entre otros, que son pigmentos industriales y, por lo tanto corresponden a intervenciones modernas.

Palabras clave: policromía, yeserías, re-policromados, mudéjar, Pedro I, Alcázar, Sevilla. interventions occurred over the original work. Natural pigments, such as red lead, cinnabar, natural azurite and malachite, were found in the deeper strata (i.e. the most likely original work). Industrial pigments, such as ultramarine blue or emerald green, were identified in the upper layers (i.e. the latest interventions).

Key words: polychrome, plasterworks, repolychrome, mudejar, medieval, Alcazar, Seville.

\section{Introducción.}

El Real Alcázar de Sevilla fue incluido en la Lista del Patrimonio Mundial de la UNESCO en el año 1987. Entre las construcciones más significativas de este espacio se encuentra el Palacio de Pedro I, edificado entre 1356-1366 ${ }^{1}$ que sin duda representa "uno de los más claros ejemplos del uso de la arquitectura como simbolización del poder del soberano" ${ }^{2 "}$.

Sus precedentes arquitectónicos son los recintos palaciegos de Tordesillas y Astudillo, mandados construir también por el monarca años antes. De ellos hay que destacar, por ejemplo, los conjuntos de yeserías que se conservan en Santa Clara de Tordesillas ya que presentan elementos comunes a las yeserías del Patio de las Doncellas como son los "motivos naturalistas y figurativos, la utilización sistemática de escritura cúfica o los atauriques de pimientos rellenos de hojas treboladas entrelazados con hojas dentadas y piñas" entre otros ${ }^{3}$.

El espectacular Patio de las Doncellas es uno de los espacios más singulares del Real Alcázar presentando "un jardín rehundido y una curiosa alberca de planta en forma de doble $T$, que pudiera estar inspirada en modelos romanos"4. Los revestimientos arquitectónicos del

${ }^{1}$ Almagro Gorbea, "Los palacios de tradición andalusí en la Corona de Castilla: Las empresas de Pedro I", p. 269.

${ }^{2}$ Almagro Gorbea, "La recuperación del jardín medieval del Patio de las Doncellas", p. 55 .

${ }^{3}$ Ruiz Souza, "Sinagogas sefardíes monumentales en el contexto de la arquitectura medieval hispana", p. 235.

${ }^{4}$ Almagro Gorbea, "Los Reales Alcázares de Sevilla", p. 178. 
patio se caracterizan por la presencia de yeserías, carpinterías, alicatados y pintura mural en la alberca. La decoración de esta zona fue alabada desde su creación por su belleza y singularidad. Se considera obra de sevillanos, toledanos y granadinos, y fruto de la amistad que unía al rey Pedro I con el sultán Muḥammad V 5 .

En este espacio destacan especialmente las yeserías y los escasos restos de policromía original en las mismas, hoy prácticamente perdida, bien por las sucesivas intervenciones de repolicromado, problemas de conservación o por los encalados que las ocultan. La riqueza del color en los revestimientos mudéjares tiene su origen en la variedad cromática que los beduinos utilizaban para contrastar la pobreza de color del desierto y que el arte musulmán transmitió al arte mudéjar ${ }^{6}$. En Irán los colores preferidos eran el azul y el verde, quizás porque inconscientemente evocaban a la vegetación y el agua. En España, el Norte de África o Egipto se desarrollan escalas diferentes en función de los materiales de los que se disponían, pero siempre en una paleta cromática a base de colores vivos ${ }^{7}$. El oro por ejemplo tomará un papel importante como símbolo de la divinidad, reservándose entre otros para los textos epigráficos del Corán, las filacterias góticas o en los emblemas heráldicos.

En resumen, la originalidad de esta decoración se debe a que combina elementos propios del arte islámico con otros cristianos, como inscripciones latinas o los símbolos de la corona en perfecta sintonía.

El Patio de las Doncellas, al ser uno de los espacios principales del palacio, ha tenido importantes intervenciones a lo largo de su historia. Entre ellas destacan las realizadas en el s. XVI que cambian completamente su fisonomía, pues se oculta el jardín rehundido mudéjar original al enlosar el patio y colocar una fuente central y se procede a la construcción de la galería superior de carácter clásico ${ }^{8}$; así como las realizadas en el s. XIX en las que se lleva a cabo reparaciones e intervenciones que podrían considerarse dentro de los presupuestos de la restauración historicista. En este sentido, las yeserías han sido partícipes de estos

\footnotetext{
${ }^{5}$ Marín Fidalgo, El Alcázar de Sevilla bajo los Austrias, 1, p. 89.

${ }^{6}$ Fernández-Puertas, "El fenómeno mudéjar en la decoración de yeserías de sus edificios", p. 191.

${ }^{7}$ González Ramírez, El trazado geométrico en la ornamentación mudéjar del Alcázar de Sevilla, p. 113.

${ }^{8}$ Morales Martínez, "El Alcázar del rey Don Pedro y los palacios mudéjares sevillanos", p. 237.
} 
cambios, sucediéndose labores de mantenimiento, readaptaciones y repolicromados sin un criterio uniforme.

El objetivo principal de esta publicación es hacer un avance de resultados del estudio de materiales, que servirá de base para un estudio más amplio de las yeserías con los tratamientos y criterios más adecuados para su conservación-restauración.

Antes de abordar una intervención de este tipo de obras es fundamental conocer las características formales y materiales, así como identificar los procesos de ejecución de las mismas. Un mayor conocimiento de nuestro patrimonio cultural conlleva un mejor mantenimiento del mismo, así como la planificación de intervenciones de conservación- restauración, con total garantía de resultados.

Este trabajo ha sido especialmente complejo por la gran cantidad de intervenciones que ha tenido. La presencia de repolicromados y encalados superpuestos ha contribuido a la pérdida de la imagen original tanto de las policromías como de su relieve ${ }^{9}$ (Fig. 1 y Fig.2).

\section{Metodología}

El trabajo de campo tenía entre otros objetivos seleccionar las zonas más representativas para la extracción de muestras.

Debido a la complejidad de la decoración y de la sucesión de intervenciones de repolicromados que se detectaron, se recogieron un número considerable de muestras. La investigación que se expone se ha basado en el análisis de un total de 129 correspondientes al paramento sur/este.

El objetivo fundamental fue identificar los principales componentes del revestimiento tanto del mortero como de las capas de policromía.

Los métodos de estudio empleados han sido:

Microscopio estereoscópico, que proporciona una primera aproximación de los materiales más allá del puro análisis visual.

\footnotetext{
${ }^{9}$ Este trabajo ha podido realizarse gracias a la subvención recibida en el Proyecto de Excelencia de la Junta de Andalucía titulado "Tratamientos cromáticos en la arquitectura de tradición musulmana. Técnica y conservación" (HUM 1941) (1/01/2014-31/12/2015) y el del Ministerio de Economía y Competitividad denominado "Decoración arquitectónica de tradición islámica. Materiales y técnicas de ejecución" (HAR 2011-27598) (01/01/201231/12/2014) así como al programa de Formación del Profesorado Universitario (FPU) del que es beneficiaria Ana Isabel Calero Castillo. De la misma manera, no hubiera sido posible sin las amabilidades de los directores del Real Alcázar de Sevilla así como a la gestión de Antonio Almagro Gorbea, de la Escuela de Estudios Árabes (CSIC) de Granada.
} 
Preparación de las muestras mediante probetas pulidas y láminas delgadas para la identificación de fases y aspectos texturales a través de microscopía óptica.

Microscopía electrónica de barrido (Scanning Electron Microscopy, SEM) mediante la obtención de imágenes de electrones secundarios retrodispersados y de microanálisis puntuales por dispersión de energía de rayos X (Energy Dispersión X-ray, EDX).

Cromatografía en fase gaseosa, para la determinación de sustancias lipófilas, como aceites secantes, resinas y ceras; y de sustancias hidrófilas, como las proteínas y las gomas así como espectroscopia IR por transformada de Fourier.

Difracción de Rayos X.

\section{Resultados}

\section{Estudio de materiales}

A partir del estudio de materiales, se obtuvo una considerable información acerca de los componentes de los estratos pictóricos y morteros. Este estudio está orientado al conocimiento de los materiales y los procesos de ejecución, lo que es fundamental para determinar el estado de conservación, las alteraciones y las intervenciones que se han sucedido a lo largo de los años.

\section{Morteros}

Aunque no es el objetivo fundamental, se ha recogido información de la técnica utilizada para la ejecución de este revestimiento en concreto. La presencia de morteros muy blancos (con un promedio de $85 \%$ de yeso) y de líneas de unión entre placas indica un procedimiento a molde en la mayoría de los casos. La analítica efectuada en 10 muestras ha evidenciado un componente mayoritario de yeso $\left(\mathrm{CaSO}_{4} 2 \mathrm{H}_{2} \mathrm{O}\right)$ que en el caso de tres muestras llegó a ser de un 100\%. En segundo lugar se ha detectado calcita $\left(\mathrm{CaCO}_{3}\right)$ como aditivo del mortero de base ${ }^{10}$. Por último, destaca la presencia de tierras de diversa procedencia. Estos

${ }^{10}$ López Cruz, García Bueno y Medina Flórez, "Evolución del color en el alero de la fachada del Rey D. Pedro I, Real Alcázar de Sevilla. Aportaciones del estudio de materiales a la identificación de las intervenciones de restauración a lo largo de su historia", p. 163-178. 
resultados son bastante parecidos a los obtenidos en el estudio de las yeserías de la Fachada de Pedro I, donde se identificó la presencia de yeso, calcita y áridos prácticamente en las mismas proporciones. Por otra parte, la combinación de procedimientos de talla y molde en la realización de este tipo de decoraciones en yeso coincide con los resultados obtenidos en otros estudios de esta tipología como los realizados en la Madraza de Granada ${ }^{11}$.

\section{Policromía}

Para estudiar la policromía se han analizado un total de 107 muestras. Como se ha comentado, las yeserías han sido muy intervenidas desde su ejecución por lo que es difícil diferenciar las zonas originales de las añadidas. El número de intervenciones se evidencia con la presencia de hasta seis estratos en algunas muestras. Generalmente mantienen la tonalidad original, aunque en ocasiones la cambian por otra diferente. La caracterización de pigmentos permite en ciertos casos identificar el periodo de aplicación (por ejemplo los pigmentos industriales), mientras que en otros, su utilización durante periodos de tiempo prolongados dificulta su interpretación ${ }^{12}$. Los acabados polícromos que se han detectado en este paramento han sido: azul, rojo, verde, amarillo y capa metálica.

Entre los pigmentos azules, destaca la presencia de azurita natural en estratos inferiores y probablemente originales ya que es un pigmento que se conoce desde la antigüedad (Fig. 3). En estratos superiores se identifica ultramar artificial. El análisis de los resultados permite concluir que el pigmento más utilizado es el ultramar artificial lo que es indicativo de que es un repinte relativamente reciente, ya que fue introducido por primera vez por Guimet en Francia en $1828^{13}$.

En los pigmentos rojos se detectó cinabrio/bermellón, rojo de plomo y tierra roja. El uso del cinabrio natural en España es bastante común desde la época romana. Su uso se atestigua desde la Antigüedad como

\footnotetext{
${ }^{11}$ García Bueno, Hernández Pablos y Medina Flórez, "Las yeserías del oratorio de la Madraza de Yúsuf I, Granada. Aportaciones de la documentación gráfica a la determinación de zonas originales y añadidos en el estudio preliminar", p. 262.

${ }^{12}$ López Cruz, García Bueno y Medina Flórez, "Evolución del color en el alero", p. 166.

${ }^{13}$ Mayer, Materiales y técnicas del arte, p. 39.
} 
manifiestan los escritos de Plinio o Teofrasto. En la producción del mineral destacan las minas de Almadén en España, considerado uno de los mejores yacimientos por Plinio, por lo que probablemente los pigmentos utilizados en el Real Alcázar procedieran de éste. La importancia de estas minas españolas está muy documentada a lo largo de la historia. En 1495 los Reyes Católicos prohibieron la entrada de productos que pertenecían "a la rama del azogue" como el bermellón para no perjudicar a las minas de Almadén y en 1498 incluso hay un caso en contra de la venta de bermellón importado de Venecia ${ }^{14}$.

Es muy significativo que en una de las muestras se identifica cinabrio en las capas inferiores y tierra roja en las superiores, lo que prueba que se mantiene la tonalidad en las intervenciones de esta zona en concreto, pero no el pigmento.

En los pigmentos verdes se detectó, malaquita en los estratos inferiores y verde esmeralda en los superpuestos. La policromía inferior realizada con malaquita se puede considerar original. Por otra parte, el verde esmeralda ya había sido identificado en la Fachada de Pedro $I^{15}$. Este verde empezó a comercializarse en 1814 por Russ e Sattler y tiene un color azul-verde intenso y luminoso bastante particular en la tonalidad, distinta al resto de colores verdes. A pesar de sus buenas cualidades, ha tenido un uso limitado entre los artistas, probablemente por su toxicidad. Hoy en día se encuentra en desuso (Fig.4) ${ }^{16}$.

Los pigmentos amarillos se reducen al empleo de amarillo de cromo, amarillo de plomo y tierra ocre. Generalmente, el amarillo de cromo y plomo no aparece de forma aislada sino asociado a capas de oro como base para su colocación al mixtión aglutinados con resinas y/o con aceites que impregnan estratos inferiores. El oro ha sido un material muy utilizado tanto en miniaturas como en pintura sobre tabla o en revestimientos. El color, la luminosidad y la inalterabilidad cuando es puro lo hacía un material de difícil sustitución. Se obtenía de monedas y cuanto mayor fuera su pureza mejor era la calidad del oro que se obtenía ${ }^{17}$. En este caso las láminas de oro son bastante puras (en torno al 100\%), aunque en un número reducido de muestras se detectan com-

${ }^{14}$ Bruquetas, "El bermellón de Almadén: de Plinio a Goya", p. 58.

${ }^{15}$ López Cruz, García Bueno y Medina Flórez "Evolución del color en el alero", pp. 163-178.

${ }^{16}$ Occorsio, "Verdi", p. 293.

${ }^{17}$ Pietropaoli y Milaneschi, "Gialli”, p. 225. 
ponentes como plata y cobre y por lo tanto de peor calidad y más susceptibles al deterioro.

\section{Aglutinantes}

El estudio de aglutinantes se efectuó sobre 12 muestras. Los resultados demostraron el empleo de cola animal en los estratos inferiores. Esto coincide con la aplicación de temples magros en obras de similar cronología, como las yeserías de la Madraza ${ }^{18}$ o el Cuarto Real de Santo Domingo ${ }^{19}$ de la ciudad de Granada.

Por otra parte, el oro se aplica al mixtión sobre una base de aceites y resinas diterpénicas a las que se añade amarillo de cromo y plomo como secativo. Este estrato amarillo constituye una base muy adecuada para el oro ya que minimiza el efecto de las lagunas en el caso de que éstas se produzcan. El empleo de amarillo de cromo lleva a concluir que estos acabados metálicos son intervenciones recientes correspondientes al siglo XIX ${ }^{20}$.

\section{Conclusiones}

Como se ha puesto de manifiesto, el estudio del paramento ha sido bastante complejo debido a las intervenciones realizadas.

De manera general se puede afirmar que la policromía aparente es consecuencia de intervenciones de restauración y adaptaciones a la moda de la época previas. En este sentido, las catas realizadas mediante métodos mecánicos y los resultados de los análisis de laboratorio han evidenciado la conservación de policromía original en estratos inferiores.

El interés principal de los resultados obtenidos es que permite llegar a hacer una aproximación a la evolución cromática de las yeserías. El

\footnotetext{
${ }^{18}$ García Bueno, Medina Flórez y González Segura, "La policromía de las yeserías del oratorio", p. 251.

${ }^{19}$ García Bueno y Medina Flórez, "The Nasrid Plasterwork at "Qubba Dar al-Manjara 1-Kubra" in Granada: Characterisation of Materials and Techniques", p. 81.

${ }^{20}$ Los análisis de aglutinantes han sido efectuados por Enrique Parra Crego, a través de la empresa LARCO QUÍMICA. Madrid.
} 
empleo de azurita, malaquita, cinabrio o rojo de plomo aglutinados con colas proteínicas en niveles de policromía inferiores induce a pensar que nos encontramos con intervenciones originales o muy cercanas a las policromías originales realizadas en el s. XIV ${ }^{21}$. Por otra parte, la presencia de pigmentos industriales como el verde esmeralda (1814), azul ultramar artificial (1828) o amarillo de cromo (1804-1809) 22 permiten datar estas intervenciones en una cronología reciente. En cuanto a las capas metálicas, la identificación en todas las muestras analizadas de un mixtión a base de amarillo de cromo y plomo permiten datar estas intervenciones en el siglo XIX o posterior, no conservándose acabados metálicos originales.

Por otra parte, se ha podido comprobar en un número considerable de muestras que el color se mantiene. Esto confirmaría las palabras de Rafael Cómez, quien al hablar de las restauraciones románticas en este tipo de monumentos afirma: "los pintores se limitaron a reproducir con mayor o menor fortuna el color medieval que veían por más que este haya chillado a las cansadas pupilas de los académicos decimonóni$\cos ^{\prime \prime 23}$.

En definitiva consideramos que este tipo de estudios pone de manifiesto la riqueza polícroma que presentaban las decoraciones en yeso. Su investigación contribuye al conocimiento de la variedad de los acabados cromáticos de la decoración hispanomusulmana, hoy prácticamente perdidos. Los resultados demuestran que en muchas ocasiones la imagen de las yeserías no se debe tanto a la pérdida de policromía, sino más bien al ocultamiento por labores de mantenimiento o alteraciones.

Igualmente manifiesta la importancia de la elaboración de estudios previos e investigaciones en profundidad del Patrimonio. Sólo de esta manera se garantizan intervenciones de conservación y restauración que respeten su historia y las circunstancias en las que los mismos se han desarrollado.

${ }^{21}$ Cómez, "Iconología de Pedro I de Castilla", p. 64.

${ }^{22}$ Kühn y Curran, "Chrome Yellow and Other Chromate Pigments", p. 190.

${ }^{23}$ Cómez, El Alcázar del Rey Don Pedro. Sevilla, p. 111. 


\section{Referencias bibliográficas}

Almagro Gorbea, Antonio. "Los palacios de tradición andalusí en la Corona de Castilla: Las empresas de Pedro I", en Actas del Symposio Internacional: El legado de Al-Andalus. El arte andalusí en los reinos de León y Castilla durante la Edad Media, Valladolid, Fundación del Patrimonio Histórico de Castilla y León, 2007, pp.245-281.

Almagro Gorbea, Antonio, "La recuperación del jardín medieval del Patio de las Doncellas", Apuntes del Alcázar de Sevilla, 5 (2005), pp. 44-67.

Almagro Gorbea, Antonio, "Los Reales Alcázares de Sevilla", Artigrama, 22 (2007), pp. 155-185.

Bruquetas Galán, Rosario, "El bermellón de Almadén: de Plinio a Goya” en Fatto d'Archimia. Los pigmentos artificiales en las técnicas pictóricas, Madrid, Ministerio de Educación, Cultura y Deporte, Subdirección General de Documentación y Publicaciones, 2012, pp. 171-181.

Cómez, Rafael, El Alcázar del Rey Don Pedro. Sevilla, Sevilla, Diputación de Sevilla, 2006.

Cómez, Rafael, "Iconología de Pedro I de Castilla", Historia. Instituciones. Documentos, 33 (2006), pp.61-80.

Fernández-Puertas, Antonio, "El fenómeno mudéjar en la decoración de yeserías de sus edificios", Miscelánea de Estudios árabes y Hebraicos, $31 / 3$ (1984), pp. 189-202.

García Bueno, Ana y Medina Flórez, Víctor Jesús, "The Nasrid Plasterwork at "Qubba Dar al-Manjara 1-Kubra" in Granada: Characterisation of Materials and Techniques", Journal of Cultural Heritage, 5 (2004), pp.75-89.

García Bueno, Ana, Medina Flórez, Víctor Jesús y González Segura, Alicia, "La policromía de las yeserías del oratorio de la Madraza de Yūsuf I, Granada. Primeras aportaciones del estudio de materiales para la localización de zonas originales y añadidos", Al-Qantara, 31/1 (2010), pp. 245-256.

García Bueno, Ana, Hernández Pablos, Ariadna y Medina Flórez, Víctor Jesús, "Las yeserías del oratorio de la Madraza de Yúsuf I, Granada. Aportaciones de la documentación gráfica a la determinación de zonas originales y añadidos en el estudio preliminar", Al-Qantara, 31/1 (2010), pp. 257-267.

González Ramírez, María Isabel, El trazado geométrico en la ornamentación mudéjar del Alcázar de Sevilla, Sevilla, Universidad de Sevilla. Consejería de Obras Públicas y Transportes, 1995.

Kühn, Hermann y Curran, Mary, "Chrome Yellow and Other Chromate Pigments", en Robert R. Feller (ed.), Artists 'Pigments. A Handbook of Their History and Characteristics, Washington, National Gallery of Art, 1985, pp.187-217.

López Cruz, Olimpia, García Bueno, Ana y Medina Flórez, Víctor Jesús, "Evolución del color en el alero de la fachada del Rey D. Pedro I, Real Alcázar de 
Sevilla. Aportaciones del estudio de materiales a la identificación de las intervenciones de restauración a lo largo de su historia", Arqueología de la arquitectura, 8 (2011), pp.163-178.

Marín Fidalgo, Ana, El Alcázar de Sevilla bajo los Austrias, Sevilla, Guadalquivir, 1990, Tomo 1.

Mayer, Ralph, Materiales y técnicas del arte, Madrid, Tursen Hermann Blume ediciones, 1993.

Morales Martínez, Alfredo José, “El Alcázar del rey Don Pedro y los palacios mudéjares sevillanos", en María del Carmen Lacarra Ducay, (ed.), Arte mudéjar en Aragón, León, Castilla, Extremadura y Andalucía, Zaragoza, Diputación de Zaragoza, 2006, pp.233-260.

Occorsio, Susanna, "Verdi" en Susanna Tocco (coord.) La fabbrica dei colori. Pigmenti e coloranti nella pittura e nella tintoria, (10 edición), Roma, Il Bagatto, 1986, pp. 253-299.

Pietropaoli, Rita y Milaneschi, Annamaria, "Gialli” en Susanna Tocco (coord.), La fabbrica dei colori. Pigmenti e coloranti nella pittura e nella tintoria, $\left(10^{\mathrm{a}}\right.$ edición), Roma, Il Bagatto, 1986, pp. 195-252.

Ruiz Souza, Juan Carlos, "Sinagogas sefardíes monumentales en el contexto de la arquitectura medieval hispana" en Memoria de Sefarad (catálogo de la exposición), Madrid, Seacex, 2002 pp.225-239.

Recibido: $17 / 07 / 2015$

Aceptado: 12/02/2016 


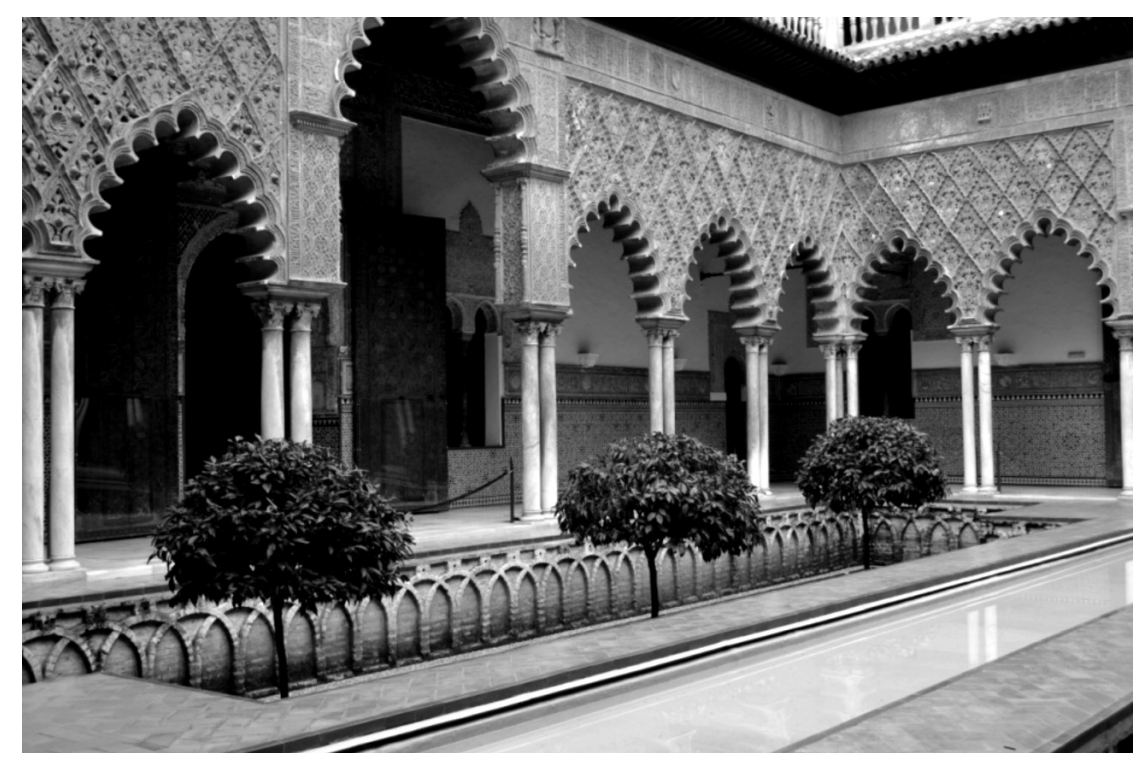

Fig, 1. Imagen general del Patio de las Doncellas. Real Alcázar de Sevilla.

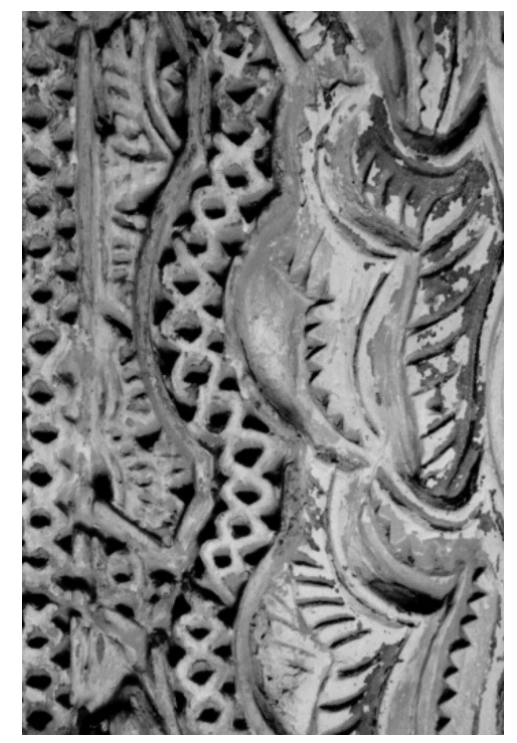

Fig.2. Imagen de detalle de las yeserías de la Puerta de Acceso al Salón del Techo de Carlos V. Primitiva Capilla de San Clemente. 


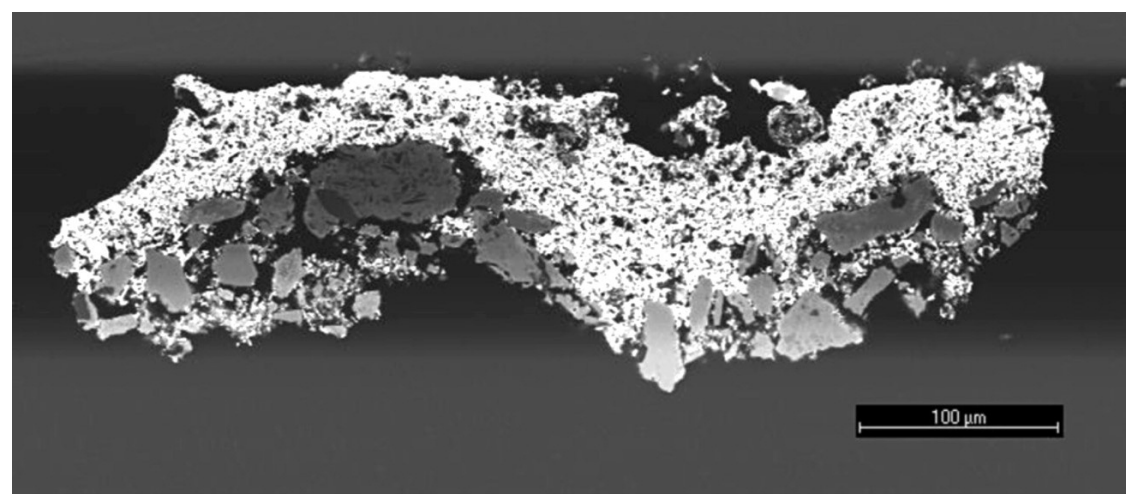

Fig. 3. Identificación de cristales de azurita en estratos inferiores de la muestra PDY40 .Imagen de SEM. Scanning Electron Microscopy.

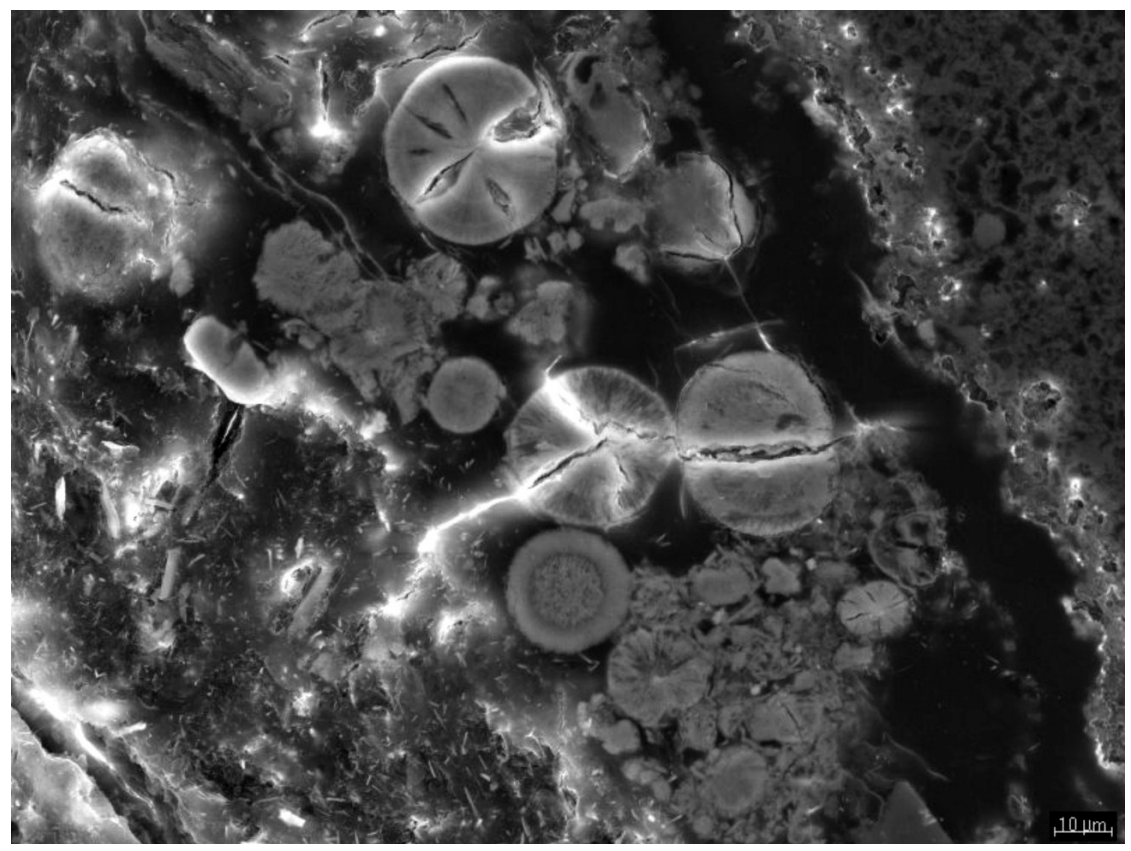

Fig. 4. Identificación del verde esmeralda a partir de sus formas esferulíticas de la muestra PDY16. Imagen de SEM. Scanning Electron Microscopy. 